\title{
Modified fingerprinting localization technique of indoor positioning system based on coordinates
}

\author{
Rhowel M. Dellosa ${ }^{1}$, Arnel C. Fajardo ${ }^{2}$, Ruji P. Medina ${ }^{3}$ \\ ${ }^{1,3}$ Graduate School, Technological Institute of the Philippines Quezon City \\ ${ }^{2}$ School of Engineering and Information Technology, Manuel L. Quezon University
}

\section{Article Info \\ Article history: \\ Received Dec 21, 2018 \\ Revised Mar 10, 2019 \\ Accepted Apr 25, 2019 \\ Keywords: \\ Brute force method Closest pair of points Received signal strength Trilateration}

\begin{abstract}
The fingerprinting localization technique is the most commonly used localization technique of the indoor positioning system. It is used by several technologies for short and long range position estimation like wireless fidelity and radio frequency. There are several schemes used to estimate a location for the indoor environment but the drawbacks resulted in complexity issues. These drawbacks have negative effects on location estimation. In order to address these drawbacks, this work attempted to explore the fingerprinting localization technique for location estimation of the indoor environment that focuses on position estimation. Results showed that the simplicity of the design of position estimation without compromising the functionality of the operations was observed with 100\% accuracy on position estimation.
\end{abstract}

\section{Copyright (0 2019 Institute of Advanced Engineering and Science.} All rights reserved.

\section{Corresponding Author:}

Rhowel M. Dellosa

Graduate School,

Technological Institute of the Philippines-Quezon City,

938 Aurora Boulevard, Cubao, Quezon City

Email: rhoweldellosa@asiatech.edu.ph

\section{INTRODUCTION}

The scene analysis of the fingerprinting localization technique is used to gather received signal strength (RSS) from the environment of concern. The RSS-based fingerprinting localization technique provides significant advantages include easy to implement, cost efficient and can be implemented in wireless fidelity and radio frequency identification technology as mentioned by [1-2]. The RSS-Based fingerprinting technique is composed of offline and online phases. The offline phase is responsible for collection and calibration of the RSS at different identified locations which are stored in a database called radio-map or fingerprint database and the online phase is responsible for capturing the RSS during operation and examines the similarity in the radio-map database to find the location whose RSS best matches the captured RSS from the mobile user with the use of positioning algorithm. Table 1 shows the advantages and disadvantages of fingerprinting localization technique as mentioned by [2-3].

Table 1. Advantages and Disadvantages of Fingerprinting Localization Technique as Mentioned

\begin{tabular}{lll}
\hline Technique & Advantage & Disadvantage \\
\hline Fingerprinting & $\begin{array}{l}\text { Improved } \\
\text { localization accuracy } \\
\text { and }\end{array}$ & $\begin{array}{l}\text { Laborious collection of } \\
\text { RSS during scene } \\
\text { analysis }\end{array}$ \\
& Easy to use & \\
\hline
\end{tabular}


The study of [3] emphasized that Channel State Information (CSI) localization technique is more robust to multipath interference and indoor noise but it is difficult for an off-the-shelf Network Interface Card which is complex hardware. The Angle of Arrival (AoA) localization technique can provide high localization accuracy without fingerprinting but needs additional antennas and complex hardware as well as algorithms. The Time of Flight (ToF) localization technique provides high localization accuracy without fingerprinting but requires synchronization between transmitter and receiver, complex hardware and antennas. This also needs a line of sight for accurate performance. The Time Difference of Arrival (TDoA) localization technique does not need any fingerprinting and does not require clock synchronization but a large bandwidth. In addition, the Return of Time of Flight (RToF) localization technique does not involve any fingerprinting but needs clock synchronization. The Phase of Arrival (PoA) localization technique can be utilized in combination with RSS, ToA, TDoA to improve overall localization accuracy but needs a line of sight to achieve high accuracy. Hence, the gaps of the localization techniques are the laborious collection and calibration of RSS stored in radio-map database, the presence of external noise interference, susceptibility to environmental change during the collection of RSS as mentioned by [2], [4-6], including the complexity of the design and availability of the resources. These gaps have negative effects on the accuracy of location estimation.

The problem of finding the closest pair of points (CPP) was applied in traffic control by [7]. It utilized the concept of CPP to monitor and contribute to the solution to traffic congestions. The most commonly used to solve the CPP program was the brute force method which requires the computation of the distances between the pair of points using Euclidean distance as used by [8-10]. The CPP is being utilized to determine the estimated position once the coordinates are already computed. In this method, there is $100 \%$ accuracy on the determination of the closest pair of points. During the determination of the closest pair of points, the number of comparisons is considered as the main factor affecting the running time of the execution. The number of comparisons after the computation of all the distances of each point to the reference point is given by (1).

$$
K=\frac{(N-1) N}{2}
$$

where:

$\mathrm{K} \quad$-Number of comparisons of the brute force method

$\mathrm{N} \quad-$ Total distance to be processed

The novel ranging method based on RSSI of [11] is a ranging method that reduces negative effects on RSSI fluctuation. It offers a small range error and higher precision compared to the traditional RSSI-based range methods. However, the complexity of the method used is still present and is in need for RSS fingerprint database which is still laborious and encounters signal attenuations. It is then followed by the unsupervised indoor localization method proposed by [12] which is used to exploit environment RSS signals including magnetic fluctuations and illumination intensity at a specific area or internal landmarks. Then again, heterogeneous hardware, phone orientation effect, energy footprint and scalability testing are some of the limitations of the study which also contribute to the robustness of the operation. In addition, the use of ZigBee to track the landmarks using the inertial navigation system (INS) and Kalman filter are also introduced by [13] which increases the accuracy of location estimation. Still, it is limited to the indoor manufacturing environment and the use of a complex algorithm for filtering of RSS may contribute a negative effect to the efficiency of the system. Another major contribution of [5] is on their locating of a fingerprint with less human intervention research which automatic labeling utilized dead reckoning by motion sensors to store data-to-radio-map database in the radio space with corresponding radio-space geometry to the physical area by actual area matching using the location path information taken from the floor plan. However, there is a need for several motion sensors to capture the RSS signal. In turn, the laborious collection of RSS is still present. In addition, the inaccuracy of generating RSS from the collection and calibration phase may also affect the performance of the localization technique such as RSS-based Fingerprinting. Some of the machines learning based algorithms for location estimation being used include maximum likelihood (ML), K-nearest neighbors (KNN), weighted KNN (WKNN), support vector machines (SVMs) and artificial neural networks (ANN). ML, KNN or WKNN are responsible for comparing gathered RSS signature with the RSS fingerprints stored in the radio map database. This is the basis of estimating the location of the object of interest mentioned by the researches of [4], [14-17]. Another technique is the use of filtering techniques on the RSS noise sources and finely tune the RSS by combining different techniques like an additional sensor data, Gaussian Mixture Model, data Dempster Shafer fusion theory of [18], fusion technique, Kalman filter of [19-22] and particle swarm filters of [23-24]. The intention of these filters is to improve the collected RSS quality on the radio map database. Nevertheless, the laborious collection of radio

Indonesian J Elec Eng \& Comp Sci, Vol. 15, No. 3, September 2019 : 1345 - 1355 
map and complexities of the system is not solved by employing filtering techniques. In addition, complexity means an increase in computational cost which affects the efficiency of position estimation. The visible light communication as presented by [25] is also possible in location estimation. In this study, light emitting diode and external sensors were used to estimate location in an indoor environment with a complex design of the antenna.

In this study, the researchers attempted to modify the fingerprinting localization technique of indoor positioning system to address the laborious collection of RSS and the complexity of the design as mentioned by [2], [4-5] and [26] which contribute variations in the result of position estimation.

\section{RESEARCH METHOD}

Three phases were considered to modify the fingerprinting localization technique. Phase 1 includes the presentation of the floor plan of the area where the indoor positioning system was implemented. Phase 2 includes the collection of RSS and the determination of the mobile coordinates. Phase 3 includes the determination of the estimated position using the brute force method.

\subsection{Phase 1 - The Floor Plan of the Area to be Monitored (Offline Phase)}

The presentation of the floorplan of the place of implementation of the indoor positioning system is depicted in Figure 1. The college library of Asia Technological School of Science and Arts was used as the place of implementation of the indoor positioning system. A scale of 1:1 meter was used.

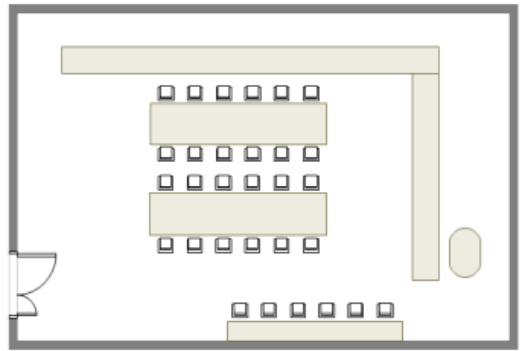

(a)

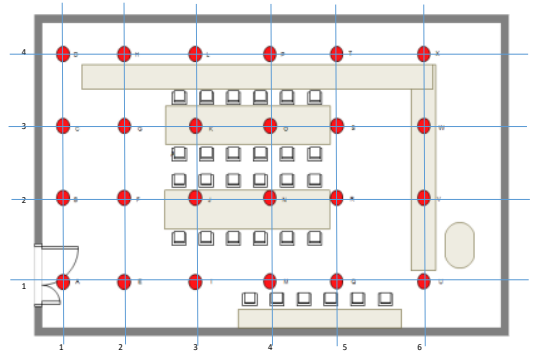

(b)

Figure 1. Floor Plan and Assigned Coordinates of College Library

\subsubsection{The Coordinates-Map}

Table 2 shows the contents of the coordinates-map table. The contents of the table were stored on the server.

\begin{tabular}{cccc}
\multicolumn{4}{c}{ Table 2. Coordinates-Map and its Locations } \\
\hline $\mathrm{P}(\mathrm{x}, \mathrm{y})$ & Location & $\mathrm{P}(\mathrm{x}, \mathrm{y})$ & Location \\
\hline 1,1 & $\mathrm{~A}$ & 4,1 & $\mathrm{M}$ \\
1,2 & $\mathrm{~B}$ & 4,2 & $\mathrm{~N}$ \\
1,3 & $\mathrm{C}$ & 4,3 & $\mathrm{O}$ \\
1,4 & $\mathrm{D}$ & 4,4 & $\mathrm{P}$ \\
2,1 & $\mathrm{E}$ & 5,1 & $\mathrm{Q}$ \\
2,2 & $\mathrm{~F}$ & 5,2 & $\mathrm{R}$ \\
2,3 & $\mathrm{G}$ & 5,3 & $\mathrm{~S}$ \\
2,4 & $\mathrm{H}$ & 5,4 & $\mathrm{~T}$ \\
3,1 & $\mathrm{I}$ & 6,1 & $\mathrm{U}$ \\
3,2 & $\mathrm{~J}$ & 6,2 & $\mathrm{~V}$ \\
3,3 & $\mathrm{~K}$ & 6,3 & $\mathrm{~W}$ \\
3,4 & $\mathrm{~L}$ & 6,4 & $\mathrm{X}$ \\
\hline
\end{tabular}

\subsection{Phase 2 - Collection of RSS}

This phase is considered the online phase wherein data collection happens. Samsung Galaxy J7 Pro was used as a mobile sensor and a Dell computer with $2.40 \mathrm{GHz}$. processor speed was used as a server. With the help of an internet connection, an android application was used to collect and store the RSS signal to the server. 


\subsubsection{Block Diagram of the RSS Data Collection}

The wireless access points were responsible for sending of RSS signals to a mobile sensor. Block diagram of data collection as shown in Figure 2.

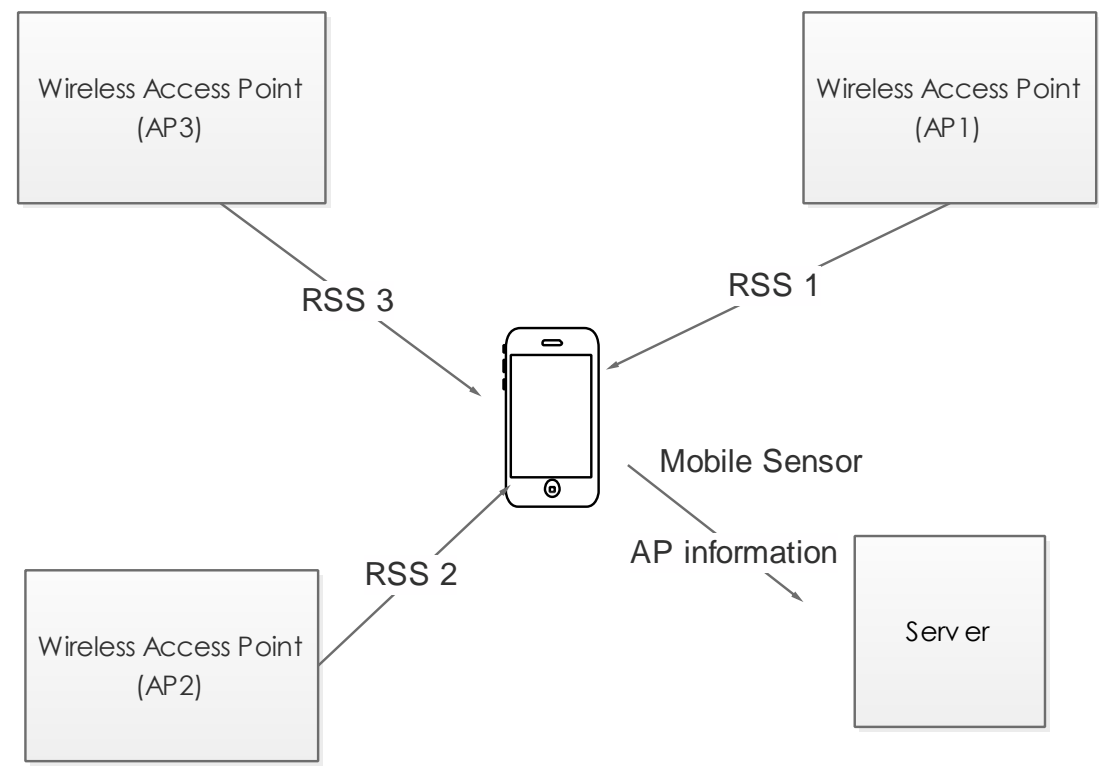

Figure 2. Block Diagram of Data Collection

\subsubsection{Mobile Coordinates Determination}

In order to determine the distances of the mobile sensor to access points, the modified Friis formula was used as provided in (2).

$$
R S S I=-10 n \log 10\left(\frac{d}{d 0}\right)+R S S I 0
$$

where:

RSSI- read-out returned by the receiver, in $[\mathrm{dBm}]$,

$\mathrm{d}$ - distance between mobile sensorand AP, in meter,

$\mathrm{n}$ - propagation constant ( 2 in free space and 4 in indoor) [2]

do- reference distance $=1$ meter

RSSI0 - RSSI read-out at reference distance d0, in [dBm].

Algebraic transformation is used to calculate the unknown distance $\mathrm{d}$ of (3), based on measured RSSI from particular mobile user, assuming the values of RSSI0 and n are known:

$$
d=10 d 0\left(\frac{R S S I 0-R S S I}{10-n}\right)
$$

In order to determine the coordinates, a geometric approach was used. Figure 3 is a trilateration technique wherein the mobile sensor and access points coordinates were illustrated.

The distance $(\mathrm{d} 1, \mathrm{~d} 2, \mathrm{~d} 3)$ can be achieved by using the concept of right triangle trigonometry as presented by the (4), (5), and (6).

$$
\begin{aligned}
& d 1^{2}=(x 1-x)^{2}+(y 1-y)^{2} \\
& d 2^{2}=(x 2-x)^{2}+(y 2-y)^{2} \\
& d 2^{2}=(x 3-x)^{2}+(y 3-y)^{2}
\end{aligned}
$$




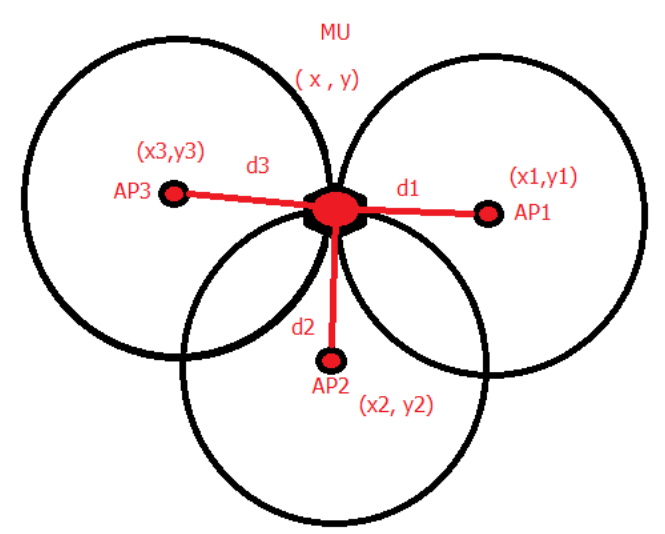

Figure 3. Geometric Illustration of mobile sensors and access points

Rearranging the (4), (5), and (6).:

$$
\begin{aligned}
& D^{2}=x 1^{2}+y 1^{2}-d 1^{2} \\
& E^{2}=x 2^{2}+y 2^{2}-d 2^{2} \\
& F^{2}=x 3^{2}+y 3^{2}-d 3^{2} \\
& x o=\frac{D(y 3-y 2)+\mathrm{E}(\mathrm{y} 1-\mathrm{y} 3)+\mathrm{F}(\mathrm{y} 2-\mathrm{y} 1)}{2[x 1(y 3-y 2)+x 2(y 1-y 3)+x 3(y 2-y 1)]} \\
& y o=\frac{D(x 3-x 2)+\mathrm{E}(\mathrm{x} 1-\mathrm{x} 3)+\mathrm{F}(\mathrm{x} 2-\mathrm{x} 1)}{2[y 1(x 3-x 2)+y 2(x 1-x 3)+y 3(x 2-x 1)]}
\end{aligned}
$$

where:

xo - x-coordinate of the object yo - y-coordinate of the object

\subsection{Phase 3-Position Estimation}

This phase includes the use of the brute force method in determining the estimated position. The computed value of coordinates was used to determine the distance of the reference point $R(i, j)$ and the set of coordinates from the coordinates-map. The following shows the flowchart of distance determination.

\subsubsection{Flowchart on How to Determine the Distance of Each Pair of Points}

Flowchart of Distance and Distance Matrix as shown in Figure 4.

\subsubsection{The Position Estimation and Sample Computation}

The matrix of distance is composed of the distances between the computed mobile sensor coordinates (xо, yo) and the set of points from the coordinates-map. Figure 5(a) presents the matrix of distance assuming a reference point $\mathrm{R}(\mathrm{i}, \mathrm{j})$ equals to $\mathrm{R}(2.3,3.2)$. The result of distance computation using the algorithm of Figure 4 is shown in Figure 5(b). Brute force method was used to compare the distances and used to determine the minimum distance. The minimum distance is necessary to determine the estimated position from the coordinates-map. Using (1), a total of 276 comparisons were needed to determine the minimum distance. The minimum distance is 0.4 which has a coordinate of $\mathrm{P}(2,3)$. Therefore, the estimated position was point $\mathrm{P}(2,3)$ at $\mathrm{G}$ position of coordinates-map. 




Figure 4. Flowchart of Distance and Distance Matrix

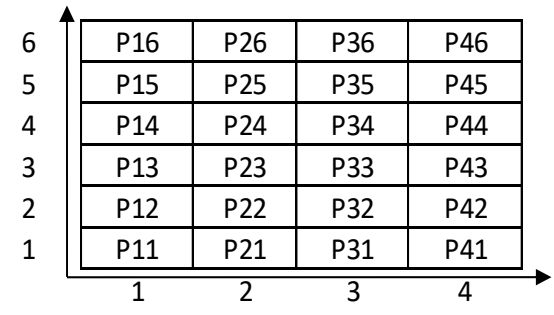

(a)

\begin{tabular}{|c|c|c|c|c|}
\hline 64 & 3.1 & 2.8 & 2.9 & 3.3 \\
\hline 5 & 2.2 & 1.8 & 1.9 & 2.5 \\
\hline 4 & 1.5 & 0.9 & 1.1 & 1.9 \\
\hline 3 & 1.3 & 0.4 & 0.7 & 1.7 \\
\hline 2 & 1.8 & 1.2 & 1.4 & 2.1 \\
\hline 1 & 2.6 & 2.2 & 2.3 & 2.8 \\
\hline
\end{tabular}

(b)

Figure 5. Matrix of Distance

\section{RESULTS AND ANALYSIS}

The following discussion presents the results of the experiments and the modification of the fingerprint localization technique. Figure 6 presents the modified fingerprinting localization technique. The modified fingerprinting localization technique starts with the collection of RSS signals up to the determination of the estimated position. The design was focused on the creation of coordinates-map and the application of brute force method on how to determine the estimated position of the mobile sensor. 


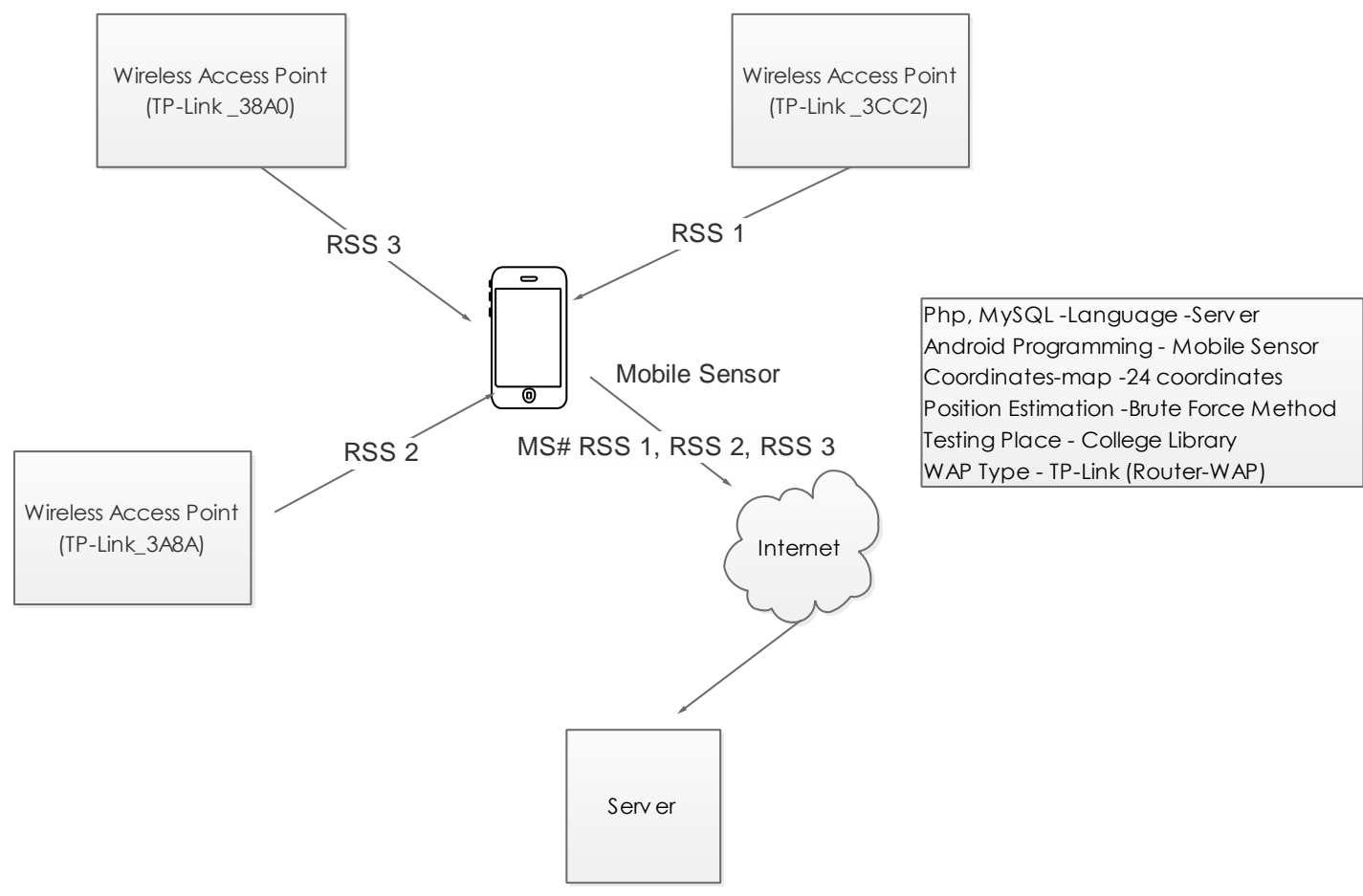

Figure 6. Modified Fingerprint Technique

\subsection{System Design}

The place of the experiment was the library of Asia Technological School of Science and Arts as presented in Figure 7(a) and 7(b).

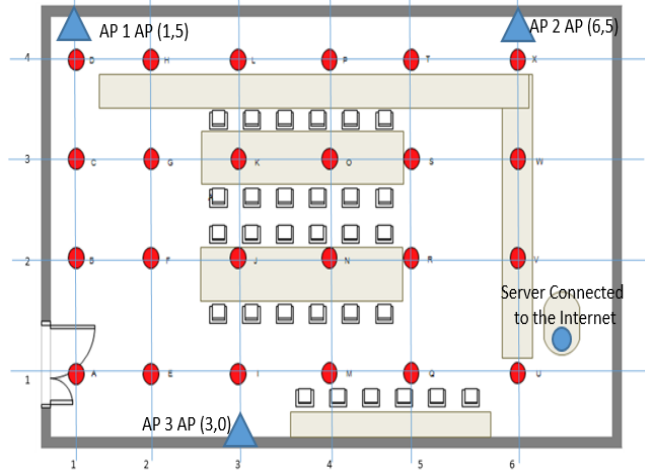

(a)

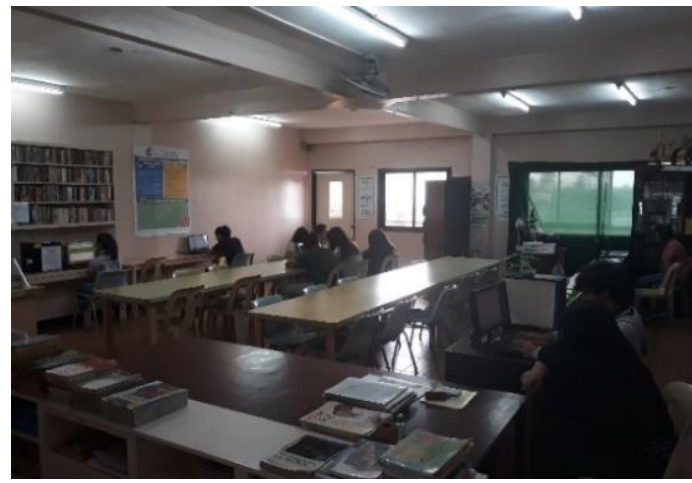

(b)

Figure 7.Wireless Access Points Set-Up and the College Library

\subsubsection{The software and development kits}

The Android software and Android development kits for mobile sensor, PHP programming language for server and MySQL server for database server were used. The Samsung Galaxy J7Pro was installed with an Android application to collect RSS and MAC address from three wireless access points. The collected RSS signals and MAC addresses were sent to the database server which was used for position estimation.

\subsubsection{The Client (Mobile Sensor)}

The Samsung Galaxy J7Pro was used as the mobile sensor that has the WiFi capabilities which are set to turn on automatically. The WiFi Manager API android application was used to scan the SSID, MAC 
address and RSS of the wireless access point. The received information by the mobile sensor is sent to the server via the internet for the position estimation process.

\subsubsection{The Server (Dell Inspiron Computer)}

The server process received information from the mobile sensor. Trilateration method was used to determine the reference point and the brute force method was used to determine the estimated position from the coordinates-map. The estimated position was also displayed on the screen of the server.

\subsubsection{Wireless Access Point Hardware Specifications}

The TP-Link TL-WR941HP model was used as a wireless access point. This wireless access point is a $450 \mathrm{Mbps}$ high power wireless $\mathrm{N}$-router and uses high power amplifiers and high gain antennas. It is capable of wireless coverage of up to 10,000 square feet and the signal from it can pass through the wall and other barriers. Table 3 summarizes the specifications of the wireless access points.

Table 3. Specifications of the Wireless Access Point

\begin{tabular}{ccc}
\hline Model & SSID & MAC Address \\
\hline TP-Link TL-WR941HP & TP-Link_3CC2 & AC-84-C6-6D-3C-C2 \\
TP-Link TL-WR941HP & TP-Link_38A0 & AC-84-C6-6D-38-A0 \\
TP-Link TL-WR941HP & TP-Link_3A8A & AC-84-C6-6D-3A-8A \\
\hline
\end{tabular}

\subsubsection{Server and Cellular Phone Hardware Specifications}

Table 4 shows the specifications of the server and the mobile sensor and Figure 8(a) and 8(b) show the wireless access point, server, the Samsung Galazy J7 Pro and the screen output of the server.

Table 4. Specifications of the Server and Mobile Sensor

\begin{tabular}{ccc}
\hline Model/Brand & CPU & Operating System \\
\hline Dell Inspiro & Intel ${ }^{\circledR}$ Core ${ }^{\mathrm{TM}} \mathrm{i} 5-6200 \mathrm{CPU} @$ 2.30Ghz. & Windows 10 \\
Samsung Galaxy J7Pro & ARM Cortex-A53, 1600Mhz, 8 Cores & Android OS 7.0 \\
\hline
\end{tabular}

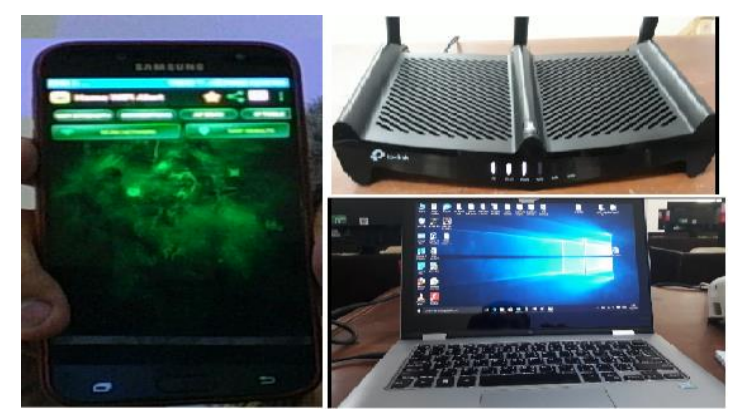

(a)

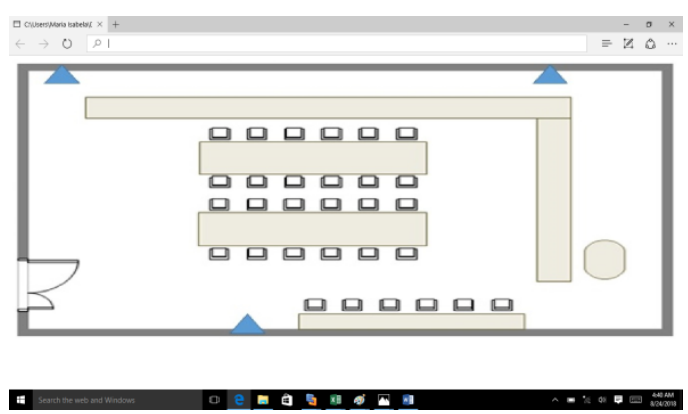

(b)

Figure 8. Android Phone, WAP and Server

\subsection{Flowchart of the Process of Modified Fingerprinting Localization Technique}

Figure 9 shows the flowchart of the process of the modified fingerprinting localization technique.

\subsection{Accuracy of the Estimated Position}

Table 5 shows several test points in the college library of Asia Technological School of Science and Arts and compared it with the location in the coordinates-map using the brute force method of comparing the points.

Based on the result of the test on accuracy in Table 5, the position estimation accuracy was best observed between 0 - 0.6 meters difference on its distances. An accuracy of $100 \%$ was observed on 10 test points with an observed distance difference ranging between 0 meters to 0.6 meters 


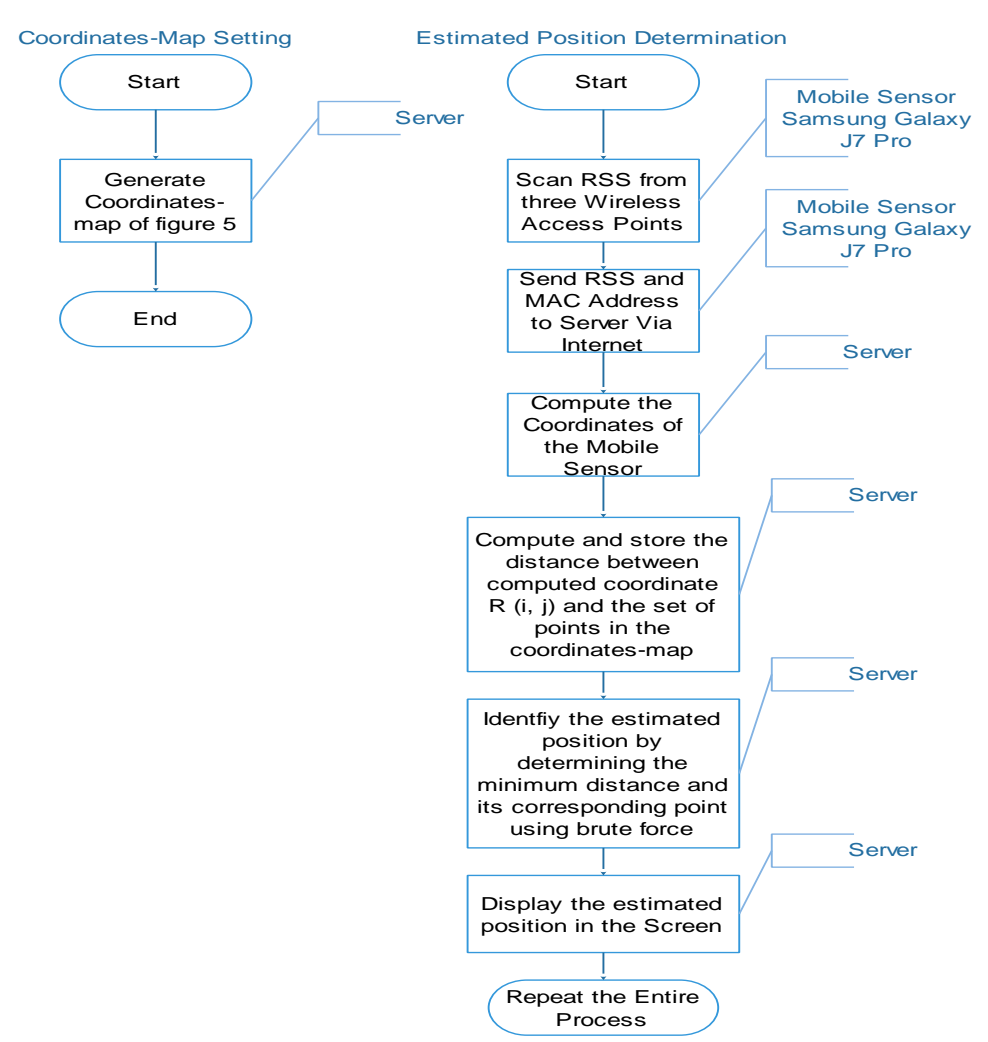

Figure 9. Process Flowchart of the Modified Fingerprinting Localization Technique

Table 5. Exact and Estimated Position

\begin{tabular}{|c|c|c|c|c|c|c|c|c|}
\hline \multirow{2}{*}{$\begin{array}{l}\text { Mobile Sensor } \\
\text { Identifier }\end{array}$} & \multirow{2}{*}{$\begin{array}{c}\text { Test } \\
\text { Point }\end{array}$} & \multicolumn{2}{|c|}{ Reference Position R (i, j) } & \multicolumn{2}{|c|}{ Estimated Position $\mathrm{P}(\mathrm{x}, \mathrm{y})$} & \multirow[t]{2}{*}{ Location } & \multirow[t]{2}{*}{ Difference } & \multirow[t]{2}{*}{ Acceptable? } \\
\hline & & $\mathrm{i}$ & $\mathrm{J}$ & $\mathrm{X}$ & $\mathrm{Y}$ & & & \\
\hline Galaxy J7Pro & 1 & 1.2 & 2.3 & 1 & 2 & $\mathrm{~B}$ & 0.4 & Yes \\
\hline Galaxy J7Pro & 2 & 1.4 & 2.6 & 1 & 3 & $\mathrm{C}$ & 0.6 & Yes \\
\hline Galaxy J7Pro & 3 & 2 & 3.2 & 2 & 3 & $\mathrm{G}$ & 0.2 & Yes \\
\hline Galaxy J7Pro & 4 & 3 & 3.4 & 3 & 3 & $\mathrm{~K}$ & 0.4 & Yes \\
\hline Galaxy J7Pro & 5 & 4 & 2.1 & 4 & 2 & $\mathrm{~N}$ & 0.1 & Yes \\
\hline Galaxy J7Pro & 6 & 3.1 & 2.1 & 3 & 2 & $\mathrm{~J}$ & 0.1 & Yes \\
\hline Galaxy J7Pro & 7 & 3.7 & 3.6 & 4 & 4 & $\mathrm{P}$ & 0.5 & Yes \\
\hline Galaxy J7Pro & 8 & 1.3 & 1.1 & 1 & 1 & A & 0.3 & Yes \\
\hline Galaxy J7Pro & 9 & 3.1 & 4 & 3 & 4 & $\mathrm{~L}$ & 0.1 & Yes \\
\hline Galaxy J7Pro & 10 & 2.1 & 2.2 & 2 & 2 & $\mathrm{~F}$ & 0.2 & Yes \\
\hline
\end{tabular}

\section{CONCLUSION}

The researchers presented a modified version of the fingerprinting localization technique of an indoor positioning system. The simplicity of modified fingerprint localization technique eliminated the laborious collection of RSS during the scene analysis and the complexity of the design as mentioned by [2], [14], [19] and [26] which contribute variations in the result of location estimation. The modification started with the determination of the floor plan of the place of implementation. The place of implementation was assigned coordinates and recorded in the database called coordinates-map. Three wireless access points were utilized to provide RSS. The mobile sensor is used to receive RSS signals from the wireless access points and send the received RSS signals to the server via the internet for storage. The MySQL database is used as the storage mechanism of the RSS and the assigned coordinates in the coordinates-map. The server is used to gather all the RSS signals from the mobile sensor and used to compute the estimated position with the aid of trilateration and brute force methods. Finally, the server will display the floorplan with the corresponding location of the mobile sensor. Future work may focus on the improvement of the matching algorithm to determine the estimated position to increase the accuracy in terms of the number of comparisons and time complexities. 


\section{ACKNOWLEDGEMENTS}

The authors would like to thank the Commission on Higher Education, Asia Technological School of Science and Arts, Technological Institute of the Philippines-Quezon City and Manuel L. Quezon University for all the support on this research endeavors. Our family, loved ones, respondents and friends who were directly and indirectly involved in this research and the Almightly God whose power is always present.

\section{REFERENCES}

[1] H. Wang, et.al, "A Novel Ranging Method Based on RSSI," Energy Procedia, vol. 12, pp. 230-235, 2011.

[2] Z. Yang, et.al, "From RSSI to CSI: Indoor Localization via Channel Response," ACM Computing Surveys (CSUR), vol. 46, no. 2, p. 25, 2013.

[3] F. Zafari, et.al, "A Survey of Indoor Localization Systems and Technologies," Cornell University Library, 2018.

[4] S. Xia, et.al,'Indoor Fingerprinting Positioning based on WiFi: An Overview. International Journal of GeoInformation, MDPI, 2017.

[5] Z. Xiao, et.al, "A Survey on Wireless Indoor Localization from the Device Perspective," ACM Computing Surveys (CSUR), vol. 49, no. 2, p. 25, 2016.

[6] J. Xiong, "Pushing the Limits of Indoor Localization in Todays Wi-Fi Networks," PhD thesis, UCL (University College London), 2015.

[7] Q. Ge, et.al, "An Improved Algorithm for Finding the Closest Pair of Points," Journal of Computer Science and Technology, vol. 21, Issue 1, pp. 27-31, Jaunary 2006.

[8] J. Jiang, Gillespie, "Engineering the Divide-and-Conquer Closest Pair Algorithm,"Journal of Computer Science and Technology, vol. 22, no. 4, pp. 532-540, 2007.

[9] Y. Zhou and H. Yu, "An Efficient Comparison-based Deterministic Algorithm to Solve the Closest Pair Problem," 2015 8th International Conference on Intelligent Computation Technology and Automation (ICICTA), Nanchang, pp. 145-148, 2015.

[10] J. C. Pereira and F. G. Lobo, "An Optimized Divide-and-Conquer Algorithm for the Closest-Pair Problem in the Planar Case,” Journal of Computer Science and Technology, vol.27, issue 4, pp.891-896, 2012.

[11] C. Tseng,et.al, "Enhanced Gaussian Mixture Model of RSSI Purification for Indoor Positioning," Journal of Systems Architecture, vol. 81, pp. 1-6, November 2017.

[12] Y. Wang, et.al, "WarpMap: Accurate and Efficient Indoor Location by Dynamic Warping in Sequence-Type Radio-Map," 2016 13th Annual IEEE International Conference on Sensing, Communication, and Networking (SECON), pp. 1-9,2016.

[13] W. Simoes, et.al "A Location Technique Based on Hybrid Data Fusion Used to Increase the Indoor Location Accuracy,” Procedia Computer Science, vol.113, pp. 368-375, 2017.

[14] C Huang and C. Chan, "ZigBee-based Indoor Location System by K-Nearest Neighbor Algorithm with Weighted RSSI," Procedia Computer Science, vol. 5, pp. 58-65, 2011.

[15] L. Jiang, “A WLAN Fingerprinting based Indoor Localization Technique,"Computer Science and Engineering: Theses, Dissertations, and Student Research. University of Nebrasca-Lincoln, 2015.

[16] Salamah, et.al, "An Enhanced WiFi Indoor Localization System based on Machine Learning," In 2016 International Conference on Indoor Positioning and Indoor Navigation (IPIN), University of Alcalá, Madrid, Spain, 2016.

[17] R. Ying, et.al, "A Simple Efficient Approximation Algorithm for Dynamic Time Warping," SIGSPATIAL'16, Burlingame, CA, USA, 2016.

[18] D. Bhatt, et.al, "A Novel Approach Towards Utilizing Dempster Shafer Fusion Theory to Enhance WiFi Positioning System Accuracy," Pervasive and Mobile Computing, vol. 37, Issue C, pp. 115-123, June 2017.

[19] X. Fang, et.al, "Noise-Aware Fingerprint Localization Algorithm for Wireless Sensor Network based on Adaptive Fingerprint Kalman Filter," Computer Network, vol. 124, Issue C, pp. 97-107, September 2017.

[20] Guvenc, "Enhancements to RSS based Indoor Tracking Systems using Kalman Flters," in GSPx \& international signal processing conference, pp. 91-102, 2003.

[21] Stanculeanu, et.al, " Enhanced RSSI Localization System for Asset Tracking Services using Non Expensive IMU," IFAC Proceedings Volumes, vol. 45, Issue 6, pp. 1838-1843, 2012.

[22] D. Zhu, et.al, "Mobile Target Indoor Tracking based on Multi-Direction Weight Position Kalman Filter,"Computer Networks, vol. 141, pp. 115-127, August 2018

[23] J. Svečko, et.al, "Distance Estimation using RSSI and Particle Filter," ISA Transaction, vol. 55, pp. 275-285, March 2015.

[24] F. Zafari, et.al, "Enhancing ibeacon based Microlocation with Particle Fltering," in 2015 IEEE Global Communications Conference (GLOBECOM), pp. 1-7, 2015

[25] M.S. Rahman, M.D. M Haque and K.D. Kim, "Indoor Positioning by LED Visible Light Communication and Image Sensors," International Journal of Electrical and Computer Engineering. Vol 1, No 2, 2011.

[26] W. Huang, "Walkielokie: Sensing Relative Positions of Surrounding Presenters by Acoustic Signals," in Proceedings of the 2016 ACM International Joint Conference on Pervasive and Ubiquitous Computing, pp. 439450, 2016. 


\section{BIOGRAPHIES OF AUTHORS}


Rhowel M. Dellosa obtained his Bachelor of Science degree in Computer Engineering from Saint Joseph Institute of Technology, Philippines in 2000. In 2002, he obtained a separate Bachelor of Science degree in Electronics and Communications Engineering from the same institution. He finished his Master's degree in Engineering with specialization in Computer Engineering in 2007 from the University of the City of Manila. He is currently taking up Doctor of Engineering with specialization in Computer Engineering from the Technological Institute of the Philippines in Quezon City, Philippines. His research interests include computational geometry, wireless sensor networks, statistical analysis and computer forensics.

Arnel C. Fajardo received his B.S. Electrical Engineering degree in 1991 from the Mapúa Institute of Technology in Intramuros, Manila. He obtained his M.S. Computer Science degree in 1999 from De La Salle University Manila. He obtained his Ph.D. in Computer Engineering degree from Hanbat National University, Daejeon, South Korea in 2014. He is the Senior Assistant Vice President and Research Director of Manuel L. Quezon University and Professorial lecturer of various universities in the Philippines. He is an author of more than 30 articles in journals and conference proceedings. His research interest includes speech recognition, image processing artificial intelligence and engineering education.

Ruji P. Medina is Dean of Graduate Programs of the Technological Institute of the Philippines in Quezon City, Philippines. He received his B.S. Chemical Engineering degree from the University of the Philippines - Diliman, Quezon City in 1992. He graduated summa cum laude from the Mapúa Institute of Technology in Intramuros, Manila in 2000 with a M.S. in Environmental Engineering degree. He holds a Ph.D. in Environmental Engineering from the University of the Philippines with a sandwich program at the University of Houston, Texas. He counts among his expertise environmental and mathematical modeling, urban mining, and nanomaterials. Apart from his active role in research and graduate engineering education, he is an excellent technical mentor with numerous publications under his name. 